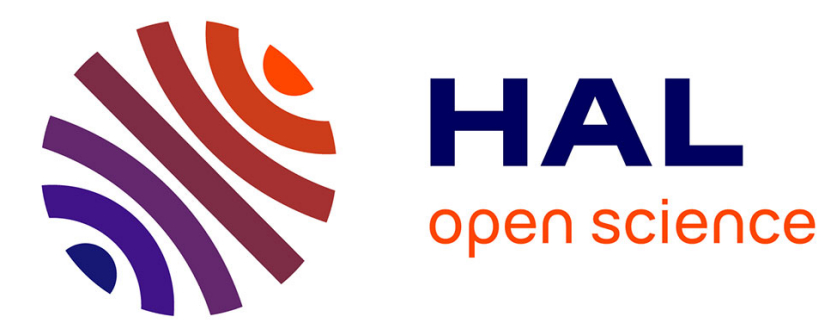

\title{
Sampling of Shape Expressions with ShapEx
}

Nicolas Basset, Thao Dang, Felix Gigler, Cristinel Mateis, Dejan Nickovic

\section{To cite this version:}

Nicolas Basset, Thao Dang, Felix Gigler, Cristinel Mateis, Dejan Nickovic. Sampling of Shape Expressions with ShapEx. MEMOCODE 2021, Nov 2021, Beijing, China. hal-03477783

\section{HAL Id: hal-03477783 \\ https://hal.science/hal-03477783}

Submitted on 13 Dec 2021

HAL is a multi-disciplinary open access archive for the deposit and dissemination of scientific research documents, whether they are published or not. The documents may come from teaching and research institutions in France or abroad, or from public or private research centers.
L'archive ouverte pluridisciplinaire HAL, est destinée au dépôt et à la diffusion de documents scientifiques de niveau recherche, publiés ou non, émanant des établissements d'enseignement et de recherche français ou étrangers, des laboratoires publics ou privés. 


\title{
Sampling of Shape Expressions
}

\author{
Nicolas Basset ${ }^{1}$, Thao Dang ${ }^{1}$, Felix Gigler ${ }^{2}$, Cristinel Mateis ${ }^{2}$, Dejan Ničković ${ }^{2}$ \\ 1 Université Grenoble-Alpes, Grenoble, France \\ 2 AIT Austrian Institute of Technology GmbH, Vienna, Austria
}

Cyber-physical systems (CPS) are increasingly becoming driven by data, using multiple types of sensors to capture huge amounts of data. Extraction and characterization of useful information from big streams of data is a challenging problem. Shape expressions facilitate formal specification of rich temporal patterns encountered in time series as well as in behaviors of CPS. In this paper, we introduce a method for systematically sampling shape expressions. The proposed approach combines methods for uniform sampling of automata (for exploring qualitative shapes) with hit-and-run Monte Carlo sampling procedures (for exploring multi-dimensional parameter spaces defined by sets of possibly non-linear constraints). We study and implement several possible solutions and evaluate them in the context of visualisation and testing applications.

\section{Introduction}

Cyber Physical Systems (CPS) are becoming increasingly driven by data. Today, typical CPS applications employ a large variety of sensors to measure, gather and analyse huge amounts of data that capture multiple aspects of the system, its physical characteristics, its internal state and operation, and its environment. The availability of this incredible amount of data enables new opportunities to improve the system operation. It requires analysing raw data, extracting and characterizing useful information from it - an extremely challenging task that has been traditionally studied by the time-series analysis in the machine learning community [37.

Shape Expressions (SE) is a specification language that facilitates the characterization of complex shapes encountered in time series [32. It is an explainable and interpretable formalism that allows to describe mean behaviors in presence of noise. SEs consist of regular expressions over atomic parametric shapes augmented with constraints on these parameters. SE specifications can be designed using the domain knowledge or can be automatically mined from raw time series. These specifications can represent an important artefact in documenting expected or observed behavioral patterns, in detecting anomalies and in understanding the overall system behavior.

We illustrate the specification of sequential behaviors in SE with a pulse train example, depicted in Figure 1. A pulse train is a sequence of pulses, where each pulse is characterized by a sequence of several segments: (A) a constant segment at some value in $(4,10)$ with duration in $(6,10),(\mathrm{B})$ a linearly decreasing segment with slope in $(-10,-1)$ and duration in $(1,4),(\mathrm{C})$ another constant segment at 0 with duration in $(2,4)$, and two consecutive linearly increasing segments, (D) 


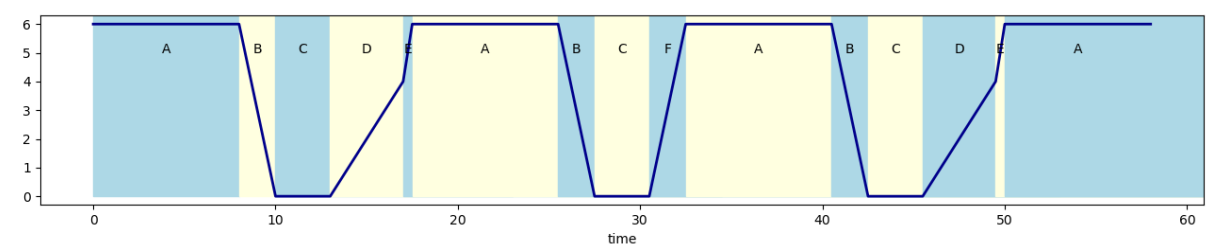

Fig. 1: Illustrating example - a pulse train.

with slope in $(1,2)$ and duration in $(0.5,2)$, and $(\mathrm{E})$ with slope in $(5,900)$ and duration in $(0.01,2)$. We finally also allow the concatenation of segments (D) and (E) to be replaced by a single segment (F), a linearly increasing segment with slope in $(1,5)$ and duration in $(2,10)$. We observe that the above specification uses parametric shapes, where the parameters are possibly constrained, and describes perfect shapes without accounting for noise. We express the pulse train specification in $\mathrm{SE}$ as follows:

$$
\begin{array}{ccc}
\varphi_{\text {pulse }}=(A \cdot B \cdot C \cdot(D \cdot E \cup F))^{+} \cdot A: \gamma, \text { where } \\
\\
A=\operatorname{lin}_{x}\left(a_{1}, b_{1}, d_{1}\right) & \gamma=a_{1}=0 \wedge b_{1} \in(4,10) \wedge d_{1} \in(6,10) \wedge \\
B=\operatorname{lin}_{x}\left(a_{2}, b_{2}, d_{2}\right) & a_{2} \in(-10,-1) \wedge d_{2} \in(1,4) & \wedge \\
C=\operatorname{lin}_{x}\left(a_{3}, b_{3}, d_{3}\right) & a_{3}=0 \wedge b_{3}=0 \wedge d_{3} \in(2,4) & \wedge \\
D=\operatorname{lin}_{x}\left(a_{4}, b_{4}, d_{4}\right) & a_{4} \in(1,2) \wedge d_{4} \in(0.5,2) & \wedge \\
E=\operatorname{lin}_{x}\left(a_{5}, b_{5}, d_{5}\right) & a_{5} \in(5,900) \wedge d_{5} \in(0.01,2) & \wedge \\
F=\operatorname{lin}_{x}\left(a_{6}, b_{6}, d_{6}\right) & a_{6} \in(1,5) \wedge d_{6} \in(2,10) & \wedge \gamma^{\prime}
\end{array}
$$

where $(a, b, d)$ denote the slope, the relative offset and the duration of a segment, respectively. In order to make sure that every segment in the sequence starts where the previous segment ends, we set $\gamma^{\prime}$ to the following constraint:

$$
\forall i \in[1,4], b_{i+1}=a_{i} d_{i}+b_{i} \wedge b_{6}=a_{3} d_{3}+b_{3} \wedge b_{1}=a_{5} d_{5}+b_{5}=a_{6} d_{6}+b_{6} .
$$

We first observe that despite an explainable syntax, the exact meaning of the specification $\varphi$ may not be self-evident. Hence, a graphical visualisation may help the designer to interpret the specification. This observation is also valid for other specification formalisms such as Signal Temporal Logic STL [29], which motivates the work on automatically generating signals satisfying STL [36.

We also note that $\mathrm{SE}$ specifications can be a valuable asset in the testing activities. Indeed, cyber-physical systems rarely operate in unconstrained environments and their testing or falsification should ideally reflect realistic environment profiles. An environment (input) profile represents a possibly infinite set of input signals subject to various constraints. It is typically obtained from the domain knowledge of the engineer, but also more recently from mining the recorded environment behavior. As an example, the pulses defined and constrained by $\varphi$ can be used as inputs from the environment to stimulate a system under test. In addition, shape expressions provide an abstraction that retains essential features of a complex dynamical component, which generates inputs to another 
component, so that the whole system can be tested in a compositional way. We will illustrate, via a control system case study, the usefulness of SE in specifying constrained behavior spaces that arise in CPS falsification and testing. The importance of constraining the input space was already observed in early work on hybrid systems falsification/testing [31. More generally, it is closely related to constrained random verification initially developed for electronic designs (see for example [40 24]), where constraints are introduced not only to ensure that the stimuli are valid or realistic (so as to avoid false negative testing results), but also to guide test executions towards critical behaviors. When there is no information about the inputs that falsify the property, it is of interest to have a more principled exploration of the input space. For these reasons, we focus on the problem of automated systematic random generation of behaviors defined by SE specifications, useful for both testing/falsification and specification visualizing.

The pulse train example exposes many challenges associated to the automated generation of behaviors from shape expressions. First, there is the problem of potentially high number of dimensions. Every line segment in $\varphi$ is uniquely characterized by its (slope, relative offset, duration) parameters. After removing parameters that do not change in the pulse example, the specification still has 11 dimensions to be explored. Second, the requirement that every segment starts where the previous one ends introduces constraints, which are both non-linear and "thin". Finally, the specification can include structural non-deterministic choices, in the form of union and Kleene star operations that need to be taken into account when sampling.

In this paper, we propose a procedure for systematic coverage-based generation of behaviors from SE specifications. We propose a two-stage sampling procedure: (1) we first choose a random word accepted by the specification with uniform distribution using the Boltzmann sampling technique [14; (2) in the second stage, we choose a random parameter valuation that satisfies the constraints using a hit-and-run [39] sampling method. Hit-and-run is a Markov Chain Monte Carlo (MCMC) sampling method that iteratively generates a sequence of points that satisfy a constraint by taking steps of (uniformly) random length in (uniformly) random directions. Hit-and-run has several desired characteristics with respect to the above identified challenges: (1) it can be used to sample arbitrary open sets in $\mathbb{R}^{n},(2)$ it asymptotically converges to uniform sampling, and (3) the complexity of the procedure is polynomial in the number of dimensions when the constraints are convex. We implement our procedure and evaluate it on multiple case studies, including visualization of ECG heart-beat specifications and test generation of inputs constrained by SE in an avionic application.

\section{Shape Expressions}

In this section, we present shape expressions (SE), a formal specification language introduced in [32]. An SE is a regular expression over parameterized atomic shapes, such as line, exponential and sinsuoid shapes. 
Let $P=\left(p_{1}, \ldots, p_{n}\right)$ be a set of parameter variables. A parameter valuation $v$ is defined as the mapping $v: P \rightarrow \mathbb{R}$. We denote $\left(v\left(p_{1}\right), \ldots, v\left(p_{n}\right)\right)$ as $v(P)$. A constraint $\gamma$ is a Boolean combination of inequalities over P. A valuation $v$ satisfies $\gamma$ (denoted by $v=\gamma$ ) iff replacing all variables $p \in P$ appearing in $\gamma$ with $v(p)$ makes $\gamma$ evaluate to true. The set of all constraints over $\mathrm{P}$ is denoted by $\Gamma(P)$. For a constraint $\gamma \in \Gamma(P)$, we denote by $\mathcal{P}(\gamma)$ the set of parameter valuations $v$ that satisfies $\gamma$, that is $\mathcal{P}(\gamma)=\{v \mid v \models \gamma\}$. Further, we assume that every constraint $\gamma$ involved in this paper yields a set $\mathcal{P}(\gamma)$ whose volume is well-defined and non-null.

Let $\mathrm{X}$ be a set of real-valued signal variables and $[0, \mathrm{~d})$ denote a time interval for some $d \in \mathbb{R}_{\geq 0}$. A signal $w$ is a function $w: X \times[0, d) \rightarrow \mathbb{R}$ that maps variables in $X$ at times in $[0, d)$ to real values. The length of $w$ is denoted by $|w|=d$. Signals with an empty time domain are permitted and result in the unique empty signal. Let $w_{1}: X \times\left[0, d_{1}\right) \rightarrow \mathbb{R}$ and $w_{2}: X \times\left[0, d_{2}\right) \rightarrow \mathbb{R}$ be signals. Their concatenation denoted by $w_{1} \cdot w_{2} \equiv w$ is then defined as

$$
w: X \times\left[0, d_{1}+d_{2}\right) \rightarrow \mathbb{R}, w(x, t)= \begin{cases}w_{1}(x, t) & \text { if } t \in\left[0, d_{1}\right) \\ w_{2}\left(x, t-d_{1}\right) & \text { if } t \in\left[d_{1}, d_{1}+d_{2}\right)\end{cases}
$$

While shape expressions can capture specifications matched by noisy signals [32, in this paper we focus on ideal signals which do not have noise.

\subsection{Shape Expressions}

Let $X$ be a set of real-valued signal variables and $P$ a set of real-valued parameters. A shape expression is an expression $\phi=\psi: \gamma$, where $\psi$ is given by the grammar

$$
\psi::=\epsilon\left|f_{x}\left(p_{1}, \ldots, p_{n}, \underline{l}\right)\right| \psi_{1} \cup \psi_{2}\left|\psi_{1} \cdot \psi_{2}\right| \psi^{*},
$$

$x \in X,\left\{p_{1}, \ldots, p_{n}\right\} \subseteq P, f_{x}\left(p_{1}, \ldots, p_{n}, \underline{l}\right)$ is an atomic shape defined by the $n$-ary function $f$ over the variable $x$ with the duration $\underline{l}$ and parameters $\left\{p_{1}, \ldots, p_{n}\right\}$ and $\gamma \in \Gamma(P)$. The symbol $\epsilon$ denotes the empty word, the operators $\cup$, $\cdot$ and * denote the classical regular expressions union, concatenation and Kleene star respectively. Hence $\psi$ is a classical regular expression over atomic shapes. The set of shape expressions over $P$ and $X$ is denoted $\Phi(P, X)$.

The regular expression $\psi$ captures the qualitative aspect of the specification, while $\gamma$ contains the quantitative information of the shape expression, meaning the global constraints imposed on its parameters. The semantics of $\psi$ follows the classical semantics of regular expressions, except for the case of an atomic shape. We say that the segment of $w$ defined by its starting and ending times $t$ and $t^{\prime}$ matches $f_{x}\left(p_{1}, \ldots, p_{n}, \underline{l}\right)$ if $\underline{l}=t^{\prime}-t$ and there exists valuation $v$ over the parameters $p_{1}, \ldots, p_{n}$ such that $w_{x}\left[t, t^{\prime}\right]=f\left(v\left(p_{1}\right), \ldots, v\left(p_{n}\right)\right)\left[0, t^{\prime}-t\right]$. A sequence of atomic shapes that satisfies $\psi$ will be called a shape word of $\varphi$ and the set of such shape words is denoted by $\mathcal{S}(\varphi)$.

A signal $w$ matches a shape expressions if (1) $w$ can be segmented into a sequence of atomic shapes $u$ satisfying the SE's regular expression, and (2) there 
exists a parameter valuation under which for each atomic shape in the sequence the observed segment and the instantiated atomic shape are the same. We also say that the signal $w$ matches $u$.

\section{Sampling Shape Expressions}

In this section, we propose a procedure for sampling signals that match a given shape expression $\varphi=\psi: \gamma$. Our sampling procedure consists of two samplers: a word sampler and a point sampler. The word sampler selects shape words $u$ that satisfy the given regular expression $\psi$ and the point sampler selects parameter valuations that satisfy the constraint $\gamma$. The point sampler is based on a hitand-run algorithm that iteratively updates candidate samples. Before describing these samplers in detail we state the properties they guarantee.

Theorem 1. 1. The word sampler (1a) samples shape words of different length; (1b) the mean length of output word can be controlled; (1c) it is uniform for every possible length of output sample, i.e. every two words of the same length have the same probability to be chosen.

2. Let $v_{k} \in \mathcal{P}(\gamma)$ denote the parameter valuation satisfying the constraint $\gamma$ sampled by the point sampler after $k$ steps. Then, for any set $U \subseteq \mathcal{P}(\gamma)$ and any given value $\epsilon>0$, there exists $K_{\epsilon}$ such that the probability that $v_{k}$ is in $U$ satisfies:

$$
\forall k \geq K_{\epsilon}\left|\operatorname{Prob}\left(v_{k} \in U\right)-\frac{\operatorname{vol}(U)}{\operatorname{vol}(\mathcal{P}(\gamma))}\right|<\epsilon .
$$

The statements (1a-c) show the probability of selecting uniformly a shape word $u$ with a given mean length. The statement (2) shows that the point sampler can sample a parameter valuation inside $\mathcal{P}(\gamma)$ with the distribution converging towards the uniform distribution. We recall here that each valuation of $\mathcal{P}(\gamma)$ leads to a noiseless signal so exploring well the set $\mathcal{P}(\gamma)$ enables one to explore well the noiseless signals matching the selected shape word. In the following we provide a description of the two samplers which includes the proof sketches of the above theorem. More concretely, the statements (1) are a direct corollary of Proposition 2, and the statement (2) is proven by the asymptotic uniformity of hit-and-run, as discussed in the end of Section 3.2. Note that instead of using the word sampler one can choose a shape word and run the point sampler to explore the set of signals that matches it.

\subsection{Sampling shape words}

We now explain our word sampler that randomly samples shape words from a shape expression following techniques known as Boltzmann sampling [14. Here we give a self-contained presentation of Boltzmann sampler for regular expressions. Before defining the Boltzmann sampling algorithm, we need the notion of generating functions. A generating function $g_{\psi}$ for a regular expression $\psi$ is defined by the series $g_{\psi}(z)=\sum_{w \in \mathcal{S}(\varphi)} z^{|w|}$. We denote by $R \operatorname{conv}\left(g_{\psi}\right)$ the convergence radius of $g_{\psi}$, that is $R \operatorname{conv}\left(g_{\psi}\right)=\sup \left\{r \geq 0 \mid \forall z<r, g_{\psi}(z)<+\infty\right\}$. 
Proposition 1. The generating function of a regular expression is a rational function. It can be computed inductively using Algorithm 1 provided the regular expression is unambiguou 1 .

Proposition 2. Let $z<R \operatorname{Ronv}(g)$. The function $p_{\psi, z}$ from $\mathcal{S}(\varphi)$ to $\mathbb{R}_{\geq 0}$ such that $p_{\psi, z}(u)=\frac{z^{|u|}}{g_{\psi}(z)}$ is a probability distribution $\left(\sum_{u \in \mathcal{S}(\phi)} p_{\psi, z}(u)=1\right)$. The mean length $N_{z}=\sum_{u \in \mathcal{S}(\phi)} p_{\psi, z}(u)|u|$ of shape words sampled according to this distribution is a rational function of $z$ that can be computed from $g_{\psi}(z)$ and its derivative $g_{\psi}^{\prime}(z)$ as follows: $N_{z}=z \frac{g_{\psi}^{\prime}(z)}{g_{\psi}(z)}$. Algorithm 2 is a Boltzmann sampler of parameter $z$, i.e. it samples a shape word distributed according to $p_{\psi, z}$.

Remark 1. To tune parameter $z$ to have mean length $N$ it suffices to find the unique root of the polynomial $N q(z)-p(z)$ between 0 and $R \operatorname{conv}\left(g_{\psi}\right)$ where $p(z)$ and $q(z)$ are polynomials such that $N_{z}=p(z) / q(z)$.

Proposition 2 proves the statement (1) of Theorem 1 since any Boltzmann sampler guarantees all three properties (1a, 1b, 1c) in this statement.

\begin{tabular}{|c|c|}
\hline $\begin{array}{lll}\text { Algorithm } & \text { 1: } & \text { Generating } \\
\text { function } g_{\psi} & & \\
\end{array}$ & $\begin{array}{l}\text { Algorithm 2: Boltzmann sam- } \\
\text { pler } B(\varphi)\end{array}$ \\
\hline Switch case & \multirow{7}{*}{$\begin{array}{l}\text { Switch case } \\
\text { 1. } \psi=\epsilon: \text { return }(\epsilon) \\
\text { 2. } \psi=a: \text { return }(a) \\
\text { 3. } \psi=\psi_{1} \cdot \psi_{2}: \text { return } B\left(\psi_{1}\right) \cdot B\left(\psi_{2}\right) \\
\text { 4. } \psi=\psi_{1} \cup \psi_{2}: \text { return } B\left(\psi_{1}\right) \text { with } \\
\text { probability } g_{\psi_{1}}(z) / g_{\psi}(z) \text { and } B\left(\psi_{2}\right) \\
\text { otherwise } \\
\text { 5. } \psi=\psi_{0}^{*}: \operatorname{return} \epsilon \text { with probability } \\
1 / g_{\psi(z)} \text { and } B\left(\psi_{0}\right) \cdot B(\psi) \text { otherwise }\end{array}$} \\
\hline 1. $\psi=\epsilon:$ return 1 & \\
\hline 2. $\psi=a: \operatorname{return} z$ & \\
\hline 3. $\psi=\psi_{1} \cdot \psi_{2}:$ return $g_{\psi_{1}} g_{\psi_{2}}$ & \\
\hline 4. $\psi=\psi_{1} \cup \psi_{2}:$ return $g_{\psi_{1}}+g_{\psi_{2}}$ & \\
\hline 5. $\psi=\psi_{0}^{*}: \operatorname{return} \unlhd^{a} 1 /\left(1-g_{\psi_{0}}\right)$ & \\
\hline & \\
\hline
\end{tabular}

Example 1. The shape expression $\psi: \gamma$ specifying the pulse train from Section 1 has its generating function $g_{\psi}(z)=\frac{z^{5}+z^{6}}{1-z^{4}-z^{5}}$ (see explanations below) and the convergence radius is 0.85667 . The mean length is $N_{z}=\frac{5+6 z-z^{4}-2 z^{5}-z^{6}}{1+z-z^{4}-2 z^{5}-z^{6}}$. So for a desired $N$ one has to find $z \in[0,0.85667)$ such that $5-N+(6-$ $N) z+(N-1) z^{4}+(2 N-2) z^{5}+(N-1) z^{6}=0$. For a mean length $N=15$, the polynomials is $-10-9 z+14 z^{4}+28 z^{5}+14 z^{6}$ and one has to instantiate $z$ with the root 0.78631 . One can also check that $z=0$ yields $N_{z}=5$ : the corresponding Boltzmann sampler always outputs the smallest shape word which is $A B C F A$.

To compute the generating function, it suffices to use the inductive definition: $g_{a}(z)=z$ for every $a \in\{A, \ldots, F\}, g_{D E \cup F}(z)=z^{2}+z$, and $g_{A B C(D E \cup F)}=z^{4}+$

\footnotetext{
${ }^{1}$ An ambiguous regular expression can be disambiguated by first translating the original expression to a deterministic finite automaton (DFA), and then translate the DFA into an unambiguous regular expression.
} 
$z^{5}$. Then, $g_{(A B C(D E \cup F))+}(z)=g_{A B C(D E \cup F)}(z) g_{(A B C(D E \cup F))^{*}}(z)=\frac{z^{4}+z^{5}}{1-z^{4}-z^{5}}$. Finally we multiply by $z$ for the concatenation of $A$ to get the formula for $g_{\psi}$.

\subsection{Sampling Constrained Parameters in Shape Expressions}

We explain the the hit-and-run algorithm that we use to sample parameter constraints in shape expressions.

Let $S \subseteq \mathbb{R}^{n}$ be a bounded open set. Let $\partial D=\left\{\left(x_{1}, \ldots, x_{n}\right) \mid \sum_{i=1}^{n} x_{i}^{2}=1\right\}$ be the set of n-dimensional unit vectors; every vector of $\partial D$ defines a direction. Algorithm 3 describes the hit-and-run method in its most abstract form and can be applied to non-linear constraints. The procedure translates a point $X_{k} \in S$ into another point $X_{k+1} \in S$ by randomly choosing a direction $\Theta_{k}$ in $\partial D$ and then randomly choosing the point $X_{k+1}$ uniformly distributed on the intersection $\Xi_{k}$ between the line crossing $X_{k}$ along the direction $\Theta_{k}$ and $S$. This procedure is repeated until some stopping criterion (based on, for instance, the sample number or computation budget) is met. Algorithm 3 has two non-trivial steps:

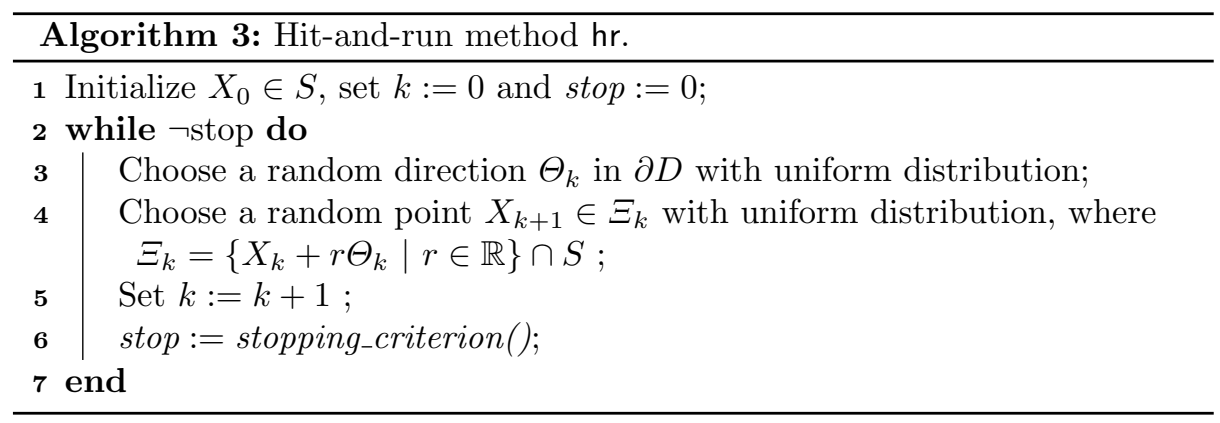

(1) the initial step of choosing $X_{0}$ in $S$, and (2) the line sampling step consisting of choosing a point in $\Xi_{k}$ with uniform distribution.

Non-linear constraints. The initialization step for arbitrary open bounded sets is done using optimization, such as particle swarm optimization (PSO) [22], an iterative optimization method that tries in every step to improve a candidate solution with regard to a given measure of quality.

The line sampling step is done by first defining an $n$-dimensional hyperrectangle $B$ that encloses $S$. Next, we intersect the line crossing $X_{k}$ along the direction $\Theta_{k}$ with $B$. The resulting line segment, denoted by $\tilde{\Xi}_{k}$, contains points that can be inside or outside $S$. We then employ 1-dimensional acceptance/rejection sampling on this line, until we get a point that is a member of $S$. Figure 2 depicts the main steps of this procedure.

Polynomial constraints. For the set $S$ defined by a set of polynomial constraints of degree $m$ in the form: $\gamma=\bigwedge_{i=1}^{k} c_{0}^{i}+c_{1}^{i} x+c_{2}^{i} x^{2}+\ldots+c_{m}^{i} x^{m}>0$, we can use a constraint solver [21] to find an initial point that lies within $S$. 

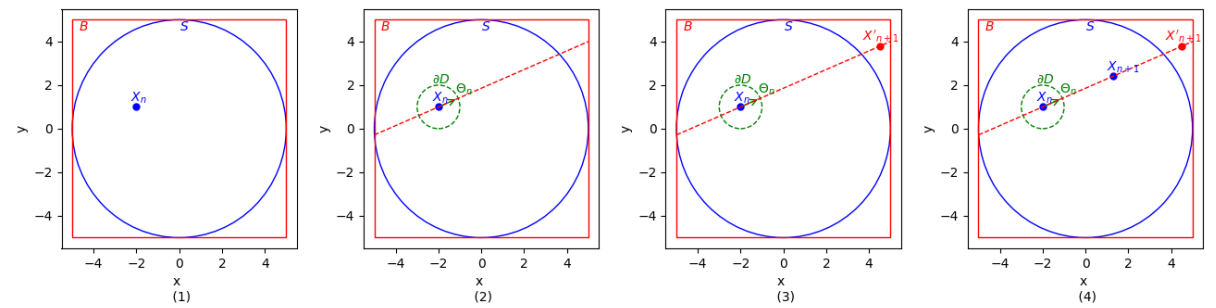

Fig. 2: An illustration of hit-and-run with the bounding hyperrectangle: (1) sample $X_{n}$ in $S$, (2) line segment $\tilde{\Xi_{n}}$ which is the intersection with $B$ of the line with random direction $\Theta_{n}$ passing through $X_{n},(3)$ rejected sample $X_{n+1}^{\prime}$ on the line segment $\tilde{\Xi_{n}}$ (such that $X_{n+1}^{\prime} \in B$ and $X_{n+1}^{\prime} \notin S$ ), and (4) accepted sample $X_{n+1}$ on the line $\left(X_{n+1} \in B, X_{n+1} \in S\right)$.

Accelerated hit-and-run. We can accelerate hit-and-run by using the so-called shrinking method [30, which works as follows: (1) let $r_{\min }=\min \left(\tilde{\tilde{\Xi}_{k}}\right)$ and $r_{\max }=\max \left(\tilde{\Xi_{k}}\right) ;(2)$ Choose a random point $r^{\prime} \in\left(r_{\min }, r_{\max }\right)$ with uniform distribution and let $X^{\prime}=\Theta_{k} r^{\prime}+X_{k} ;(3-a)$ if $X^{\prime} \in S$, then $X_{n+1}=X^{\prime}$; (3-b) if $X^{\prime} \notin S$, then: (3-b-I) if $r^{\prime}<0$, then $r_{\min }=r^{\prime}$; (3-b-II) if $r^{\prime} \geq 0$, then $r_{\max }=r^{\prime}$; (3-b-III) goto step (2).

Hit-and-run with shrinking has the same theoretical mixing time as without shrinking for convex sets, for non-convex sets it is not known. But empirically, the efficiency of hit-and-run with shrinking is superior 23 .

Coordinate directions hit-and-run. The mixing time of hit-and-run can be further acceleratedby using the coordinate directions hit-and-run (CDHR) 39] variant. In CDHR, a different direction set $\Theta_{k}$ is used, namely the set of axisaligned unit vectors. In essence, the new direction goes along one of the (randomly chosen) dimensions. This introduces a trade-off between potentially more mixing steps but simpler computation of the intersection with the bounding box. CDHR is also shown to converge to the uniform distribution asymptotically [39]. CDHR outperforms in practice the classical hit-and-run, especially in presence of parameters with different scales.

Asymptotically uniform distribution of noiseless signals for a fixed shape word. The statement (2) of Theorem 1 about the asymptotically uniform distribution of sampled noiseless signals for a fixed shape word is guaranteed by the fact the hit-and-run algorithm defines a Markov chain on the set $S$ that has a uniform stationary distribution on $S$. This means that if we sample sufficiently long, the distribution of the sampled parameters converges to the uniform distribution on $S$. Concerning the mixing time of hit-and-run, if the set is convex, it is proven to be polynomial [28]. If this set is non-convex, the mixing time is polynomial under some condition of smoothness and curvature of the set [1]. 
These results can be directly applied to our sampling procedure, depending on the constraints in the shape expressions of interest.

\section{Case Studies and Experimental Results}

We evaluate our sampling approach on several examples, including a visualization of an ECG specification and testing an aircraft elevator control system (AECS) in which we use SE to constraint inputs. We implemented a prototype sampler in Python 3.6. We used the Pyswarm library for PSO ${ }^{2}$, and $\mathrm{Z}^{3}$ 10 for SMT solving. All the experiments were carried out on a computer with the Intel Core I7-8650U processor with 4 cores, 16 Gb RAM and Windows 10 Professional operating system.

\subsection{Visualisation of Shape Expressions}

In this section, we demonstrate the use of shape expression sampling to visualize and interpret specifications. We consider visualizations of two specifications: (1) the pulse specification with polynomial constraints and a complex regular expression structure, and (2) a heart beat specification inspired from ECG signals that has thin non-linear constraints.

Figure 3 depicts 10 samples of the $\varphi_{\text {pulse }}$ behavior generated by our sampler. We observe both qualitative (number and shape of pulses) and quantitative (different parameters) variations of generated behaviors. We note that $\varphi_{\text {pulse }}$ has equality constraints, which cannot be handled by hit-and-run. We relax equality constraints by introducing an $\epsilon$ approximation.

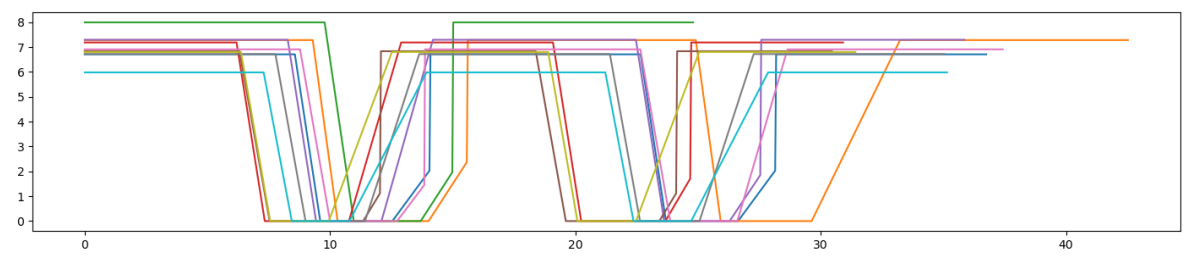

Fig. 3: Visualizing the possible behaviors of the pulse shape expression with complex regular expressions structure.

For the heart beat example, we are inspired by ECG signals from PhysioBank [18, a database containing 549 medical records including ECG readings from 209 male and 81 female subjects, aged from 17 to 87 . The database covers various diagnostic conditions related to cardio-vascular diseases.

2 https://pythonhosted.org/pyswarm/

3 https://github.com/Z3Prover/z3 
In this experiment, we manually define an SE specification for heart-beats inspired by the ECG from the database. This example illustrates visualisation of a specification that combines exponential and linear shapes. This non-linear aspect makes it interesting for exploring the visualization application. The heartbeat specification is formalized as follows: $\varphi_{\text {heart }}=A \cdot B \cdot C \cdot D \cdot E \cdot F \cdot G: \gamma$, where

$$
\begin{array}{ll}
A=\exp _{x}\left(a_{1}, b_{1}, c_{1}, d_{1}\right) \quad \gamma=a_{1}=0 \wedge b_{1} \in(0.008,0.027) \wedge c_{1} \in(30,32) \wedge \\
& d_{1} \in(0.046,0.047) \wedge \\
B=\exp _{x}\left(a_{2}, b_{2}, c_{2}, d_{2}\right) \quad & a_{2} \in(0.03,0.1) \wedge b_{2} \in(0.08,0.23) \wedge c_{2} \in(-35,-32) \wedge \\
& d_{2} \in(0.101,0.102) \wedge a_{1}+b_{1} \cdot e^{c_{1} \cdot d_{1}}=a_{2}+b_{2} \wedge \\
C=\operatorname{lin}_{x}\left(a_{3}, b_{3}, d_{3}\right) & a_{3} \in(22,30) \wedge b_{3} \in(-0.12,0.01) \wedge d_{3} \in(0.03,0.031) \wedge \\
& a_{2}+b_{2} \cdot e^{c_{2} \cdot d_{2}}=b_{3} \wedge \\
& a_{4} \in(-50,-30) \wedge b_{4} \in(0.7,0.8) \wedge d_{4} \in(0.027,0.028) \wedge \\
& a_{3} \cdot d_{3}+b_{3}=b_{4} \wedge \\
E=\operatorname{lin}_{x}\left(a_{4}, b_{4}, d_{4}\right) & a_{5} \in(20,30) \wedge b_{5} \in(-0.4,-0.3) \wedge d_{5} \in(0.012,0.013) \wedge \\
E=\operatorname{lin}_{x}\left(a_{5}, b_{5}, d_{5}\right) & a_{4} \cdot d_{4}+b_{4}=b_{5} \wedge \\
F=\exp _{x}\left(a_{6}, b_{6}, c_{6}, d_{6}\right) \quad & a_{6} \in(-0.05,0.03) \wedge b_{6} \in(0.018,0.043) \wedge c_{6} \in(8,9) \wedge \\
& d_{6} \in(0.15,0.1525) \wedge a_{5} \cdot d_{5}+b_{5}=a_{6}+b_{6} \wedge \\
G=\exp _{x}\left(a_{7}, b_{7}, c_{7}, d_{7}\right) \quad & a_{7} \in(-0.02,0.123) \wedge b_{7} \in(0.0395,0.0415) \wedge \\
& c_{7} \in(-35,-34) \wedge d_{7} \in(0.046,0.047) \wedge \\
& a_{6}+b_{6} \cdot e^{c_{6} \cdot d_{6}}=a_{7}+b_{7} .
\end{array}
$$

Due to the non-linearity of the constraints, we can only employ the hitand-run variant that uses optimization to find the initial point satisfying the constraints. We also remark that the constraints of the specification are thin. We compare the performance between the classical and the coordinate direction hit-and-run to generate samples and show the results in Figure 4 . In each case we generated 100 behaviors using 1000 mixing steps. The classical hit-andrun algorithm required $193 \mathrm{~s}$ to generate these behaviors with $0.36 \%$ acceptance rate, while the coordinate direction version of the procedure required $196 \mathrm{~s}$ with $0.14 \%$ acceptance rate. We can observe that the coordinate direction variant of the algorithm achieves much better mixing with the same number of sampled behaviors.

\subsection{Testing an Aircraft Elevator Control System with Shape Expressions and Signal Temporal Logic}

In this section, we demonstrate our approach of sampling shape expressions for systematic testing of the Aircraft Elevator Control System (AECS) [17, a CPS Simulink model that illustrates model-based design of Fault Detection, Isolation and Recovery (FDIR) in control systems by using actuation redundancy.

AECS has two elevators, on the left and one on the right side. Both elevators are equipped with two hydraulic actuators. Each actuator can position the elevator. Formal verification of CPS models such as AECS comes at a prohibitive cost. Even the medium-size AECS model represents a real challenge for exhaustive verification, with 426 real-valued, Boolean and enumerated (state) variables. 


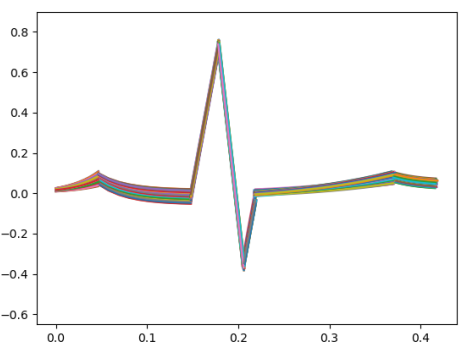

(a) classical hit-and-run

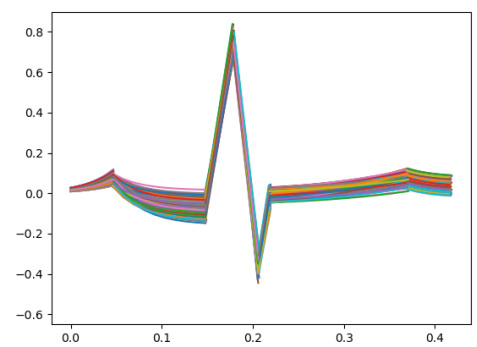

(b) coordinate direction hit-and-run

Fig. 4: Behaviors generated from $\varphi_{\text {heart }}$.

This situation favors more pragmatic simulation-based testing. This a priori incomplete verification activity can be made more principled by integrating formal specifications to express desired properties.

Signal Temporal Logic (STL) 29] is a popular declarative language for specifying CPS requirements. STL admits quantitative semantics 16]12 that allows defining the robustness degree $\rho(w, \psi)$ of a STL specification $\psi$ to a (simulation) trace $w$, that is it measures how far $w$ is from satisfying or violating $\psi$. Falsification-based testing [31] is a popular approach that uses the robustness degree to steer the CPS model to the specification violation.

A typical system-level property of AECS requires that the intended position of the aircraft given by the pilot shall be achieved within a predefined time window and with a predetermined accuracy. This can be captured with several requirements. One requirement states that when the Pilot Command $p c$ has a derivative higher than some threshold $m$, the left actuator position lep measured by the sensor must stabilize (become at most $n$ units away from the pilot command input) within $T+t$ time units (for some $t$ and $T$ ). We formalize this requirement as the following STL specification:

$$
\psi_{s t a b} \equiv \square\left(\left(p c^{\prime} \geq m\right) \rightarrow \diamond_{[0, T]} \square_{[0, t]}(|p c-l e p| \leq n)\right) .
$$

with $m=10, T=300, t=50$ and $n=0.1$.

We evaluate the following scenario - principled testing of a system-undertest (AECS model) guided by its expected properties formalized in the STL specification language (formula $\psi_{\text {stab}}$ ) under the constrained input space given by a shape expression (specification $\varphi_{\text {pulse }}$ ). In particular, we show how to use our shape expression sampling approach to improve: (1) simulation-based sensitivity analysis of the model, and (2) falsification-testing of the model.

We assume that the input (pilot command) signal is constrained with the shape expression $\varphi_{\text {pulse }}$ from the introduction. Figure 5 depicts the pilot command input (in red) from Figure 1 , and the resulting left elevator position output (in blue) obtained by executing the model in Simulink. 


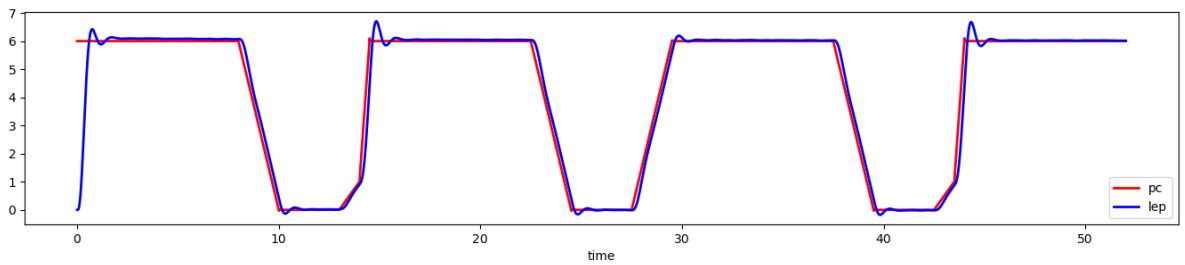

Fig. 5: The pulse train as pilot command input to AECS (red) and the resulting left elevator position (blue).

In the first experiment, we generate 50 sampled behaviors from the shape expression $\varphi_{\text {pulse }}$. To improve the uniformity of the samples' distribution, we adopt two orthogonal approaches: (1) hit-and-run with coordinate direction, and (2) accepting on average only every $100^{\text {th }}$ sample. We simulate the AECS model with each of the generated input behaviors and compute robustness with respect to the STL requirement $\psi_{\text {stab }}$. We then study how individual parameters from $\varphi_{\text {pulse }}$ affect the robustness of $\psi_{\text {stab }}$. Figure 6 shows the results for parameters $b_{0}, a_{2}, a_{4}$ and $a_{5}$. We can see that the offset $b_{2}$ at which the pulse starts and the steepness of the slope $a_{2}$ of the second segment in the pulse significantly affect the resulting robustness. The individual role of parameters $a_{4}$ and $a_{5}$ is much less clear.
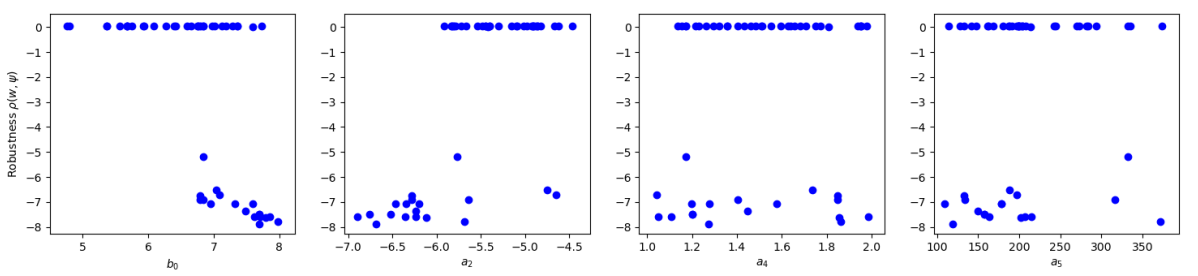

Fig. 6: Sensitivity with respect to robustness for parameters $b_{1}, a_{2}, a_{4}$ and $a_{5}$.

In the next experiment, we consider the falsification problem for the formula $\psi_{\text {stab }}$ with constrained inputs:

$$
\min _{w} \rho\left(w \| F(w), \psi_{\text {stab }}\right) \text { subject to } w \in S,
$$

where $S$ is the set of constrained inputs, $w \| F(w)$ is the combined input $(w)$ and output $(F(w))$ behavior of the AECS model, $\psi_{\text {stab }}$ is an STL formula and $\rho$ is the STL robustness function.

We first study the problem where $S$ is a simple hyper-rectangle, i.e. every input is bounded by an interval. We define the input behavior with 5 control points, each having a timestamp and a value. The value of each control point 
is in $[0,8]$ and the distance between consecutive control points is in $[0.1,10]$. This scenario reflects the common use case of falsification testing, where inputs are represented as sequences of control points. We solve the above optimization problem with Pyswarm PSO library with swarm size 10, the maximum number of iterations for the swarm to search 10 , particle velocity scaling factor 0.5 , and the scaling factor to search away from the particle's best position 0.5. After 20 simulations and $94 \mathrm{~s}$, the procedure finds an input sequence $u$ that steers the model to the violation of $\psi_{\text {stab }}$ with negative robustness of -6.57 . The input behavior, and its associated output are shown in Figure 7. We can appreciate that the input behavior does not correspond to a realistic pilot command.

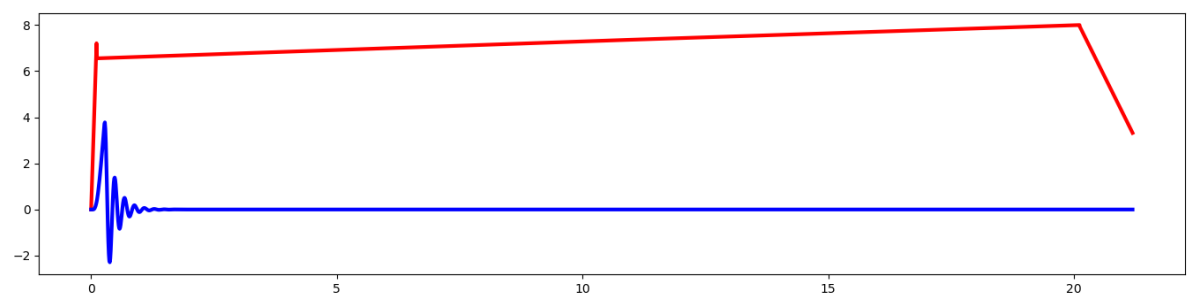

Fig. 7: Input behavior and its associated output witnessing the violation of $\psi_{s t a b}$.

We next consider the scenario where $S$ is defined by the pulse-train shape expression $\varphi_{\text {pulse }}$. We can solve in theory this optimization problem in a straightforward manner that does not require shape expression sampling. Pyswarm PSO library allows restricting inputs with arbitrary constraints. In this case, we encode the input as a set of parameters that uniquely represent the given shape expression. We then enrich the PSO procedure with a function which evaluates the generated input against the constraints from $\varphi_{\text {pulse }}$. PSO uses a rejection procedure that only applies inputs satisfying the constraints from $\varphi_{\text {pulse }}$. We ran the PSO procedure with the same parameters as above. The algorithm could not find an input that leads to the property violation after $353 \mathrm{~s}$ and 102 model simulations. We suspect that this straightforward procedure fails because it uses a trivial rejection sampling strategy. Given that the input space is both manydimensional and "thin", the procedure fails to find a point within the constraints.

Finally, we implement a procedure for falsification-testing with inputs constrained by a shape expression that incorporates the hit-and-run method. In every iteration, it uses hit-and-run to generate a new candidate input that is guaranteed to satisfy the shape expression. This input behavior is used to simulate the model, and the outcome is checked for robustness. The procedure updates the current best candidate only if it improves the robustness. In this experiment, we were able to find the input behavior that both satisfies the shape expression $\varphi_{\text {pulse }}$ and steers the model to violate $\psi_{\text {stab }}$ in 10 iterations after $32 s$. Figure 8 shows the input and its associated output behavior. The reader can 
appreciate the quality of the input with respect to the unconstrained input from Figure 7

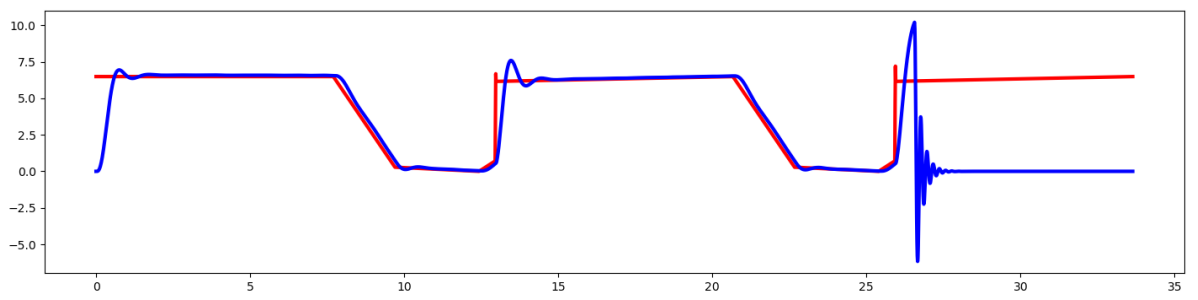

Fig. 8: Pulse train that steers AECS to specification violation.

\section{Related Work}

The hit-and-run sampling algorithm was first introduced in [39], where the author showed that the algorithm generates samples of a bounded set that are asymptotically uniform. The shrinking method that enables better convergence in the rejection sampling of a line was introduced in [30] and applied to hit-andrun in 23]. The major steps of these methods, formulated in these papers in an abstract manner, are efficiently concretized and adapted in our work for sampling points in (possibly non-convex) semi-algebraic sets, which together with the Boltzmann sampling allows systematically generating signals from shape expressions with asymptotically uniform distribution.

Hit-and-run was previously exploited by the formal methods community for falsification of hybrid systems [31, the focus of which is on using quantitative semantics of temporal specifications to guide the system-under-test towards the violation of the property, where hit-and-run method is proposed when exploring convex input domains. Note that the input domains in this work are subsets of $\mathbb{R}^{n}$. More recent work on falsification of hybrid and cyber-physical systems extends inputs to sets of signals described by piecewise functions encoded using a finite number of bounded parameters. The literature on CPS falsification and testing has become vast, we include here only some most recent papers [13 15 41445] and more references can be found therein as well as in the papers and websites related to the major state-of-the-art tools S-TaLiRo 38 and Breach 11. Compared to this approach, our work can deal with more general signals described by complex constraints involving both time and value domains. Along this line, the closest work to ours is 4 where the temporal patterns of input signal spaces are constrained by timed automata which entails particular polytopic constraints that are nevertheless simpler than the constraints yielded by shape expressions. Uniform sampling of traces satisfying given constraints has been considered in constrained random verification, for different specification and 
modeling formalisms, such as uniform and nearly-uniform sampling satisfying assignments of Boolean formulas $8 / 7 / 20$, uniformly sampling traces of networks of automata [6], timed automata [3, transition systems [9]. Monte-Carlo LTL model-checking introduced in [19] was enhanced with a uniform version [33. Uniform sampling can be used as a means of providing theoretical guarantees for random testing of distributed programs 34]. Note that our sampling method uses Boltzmann sampling, as in [6], for the discrete part (namely shape words) of shape expressions. However, unlike all the above-mentioned work, our work can deal with both temporal and spatial constraints of cyber-physical signals. As mentioned in the introduction, generating cyber-physical behaviors from STL was proposed in [36, where STL specifications are encoded as linear arithmetic real formulas and an SMT solver is used to find satisfying assignments in the form of signal behaviors. In contrast to our approach, this work does not aim at sampling specifications according to any specific distribution.

In the spirit of using randomness for exploration, statistical model-checking SMC, which is based on sampling behaviors of a system model and using statistical inference to estimate the probability that the system satisfies a property, has been applied for CPS (see some recent surveys [25|26|2|27/35] which include descriptions of the existing SMC tools). Unlike the concern of this approach involving rare events which is addressed by techniques such as importance sampling, cross-entropy methods, trace uniformity requirements have not been explored. They can be additionally considered to provide coverage guarantees especially useful when there is no information about falsifying behaviors as well as when no falsifying behaviors are revealed by the validation process.

\section{Conclusions and Future Work}

We presented an approach for generating random behaviors from shape expressions that are asymptotically uniform. It combines algorithms that were originally studied in the combinatoric community (Boltzmann sampling) and in operation research (hit-and-run sampling), exploring different variants of these procedures. We thus provided an effective approach for sampling sophisticated temporal specifications and demonstrated its usefulness for the visualisation and interpretation of formal specifications, as well as for systematic CPS testing.

We plan to study sampling of shape expressions in the presence of equality constraints. In this work, equality constraints were replaced by "thin" open constraints which significantly increase the mixing times needed to achieve almostuniformity of samples. While we tackled this problem with coordinate direction variant of hit-and-run, there is room for improving the methods that would allow faster convergence. In this paper, we used shape expressions together with the hit-and-run algorithm to narrow the input exploration space. We plan to improve the interaction between optimization engines and the shape expressions sampler to improve falsification testing. 


\section{References}

1. Yasin Abbasi-Yadkori, Peter L. Bartlett, Victor Gabillon, and Alan Malek. Hitand-run for sampling and planning in non-convex spaces. In Aarti Singh and Xiaojin (Jerry) Zhu, editors, Proceedings of the 20th International Conference on Artificial Intelligence and Statistics, AISTATS 2017, 20-22 April 2017, Fort Lauderdale, FL, USA, volume 54 of Proceedings of Machine Learning Research, pages 888-895. PMLR, 2017.

2. Gul Agha and Karl Palmskog. A survey of statistical model checking. ACM Transactions on Modeling Computing and Simululation, 28(1):6:1-6:39, 2018.

3. Benoît Barbot, Nicolas Basset, Marc Beunardeau, and Marta Kwiatkowska. Uniform sampling for timed automata with application to language inclusion measurement. In Quantitative Evaluation of Systems - 13th International Conference, QEST 2016, Quebec City, QC, Canada, August 23-25, 2016, Proceedings, pages 175-190, 2016.

4. Benoit Barbot, Nicolas Basset, and Thao Dang. Generation of signals under temporal constraints for cps testing. In Nasa Formal Methods NFM 2019, Lecture Notes on Computer Science. Springer, 2019.

5. Benoît Barbot, Nicolas Basset, Thao Dang, Alexandre Donzé, James Kapinski, and Tomoya Yamaguchi. Falsification of cyber-physical systems with constrained signal spaces. In Ritchie Lee, Susmit Jha, and Anastasia Mavridou, editors, NASA Formal Methods - 12th International Symposium, NFM 2020, Moffett Field, CA, USA, May 11-15, 2020, Proceedings, volume 12229 of Lecture Notes in Computer Science, pages 420-439. Springer, 2020.

6. Nicolas Basset, Jean Mairesse, and Michèle Soria. Uniform sampling for networks of automata. In 28th International Conference on Concurrency Theory, CONCUR 2017, September 5-8, 2017, Berlin, Germany, pages 36:1-36:16, 2017.

7. Supratik Chakraborty, Daniel J. Fremont, Kuldeep S. Meel, Sanjit A. Seshia, and Moshe Y. Vardi. On parallel scalable uniform sat witness generation. In Christel Baier and Cesare Tinelli, editors, Tools and Algorithms for the Construction and Analysis of Systems, pages 304-319, Berlin, Heidelberg, 2015. Springer Berlin Heidelberg.

8. Supratik Chakraborty, Kuldeep S. Meel, and Moshe Y. Vardi. A scalable and nearly uniform generator of SAT witnesses. In Computer Aided Verification - 25th International Conference, CAV 2013, Saint Petersburg, Russia, July 13-19, 2013. Proceedings, pages 608-623, 2013.

9. Supratik Chakraborty, Aditya A. Shrotri, and Moshe Y. Vardi. On uniformly sampling traces of a transition system. Proceedings of the 39th International Conference on Computer-Aided Design, Nov 2020.

10. Leonardo Mendonça de Moura and Nikolaj Bjørner. Z3: an efficient SMT solver. In Tools and Algorithms for the Construction and Analysis of Systems, 14th International Conference, TACAS 2008, Held as Part of the Joint European Conferences on Theory and Practice of Software, ETAPS 2008, Budapest, Hungary, March 29April 6, 2008. Proceedings, pages 337-340, 2008.

11. Alexandre Donzé. Breachflows: Simulation-based design with formal requirements for industrial CPS (extended abstract). In Sebastian Steinhorst and Jyotirmoy V. Deshmukh, editors, 2nd International Workshop on Autonomous Systems Design, ASD 2020, March 13, 2020, Grenoble, France (Virtual Conference), volume 79 of OASICS, pages 5:1-5:5. Schloss Dagstuhl - Leibniz-Zentrum für Informatik, 2020. 
12. Alexandre Donzé and Oded Maler. Robust satisfaction of temporal logic over real-valued signals. In Formal Modeling and Analysis of Timed Systems - 8th International Conference, FORMATS 2010, Klosterneuburg, Austria, September 8-10, 2010. Proceedings, pages 92-106, 2010.

13. Tommaso Dreossi, Alexandre Donzé, and Sanjit A. Seshia. Compositional falsification of cyber-physical systems with machine learning components. J. Autom. Reason., 63(4):1031-1053, 2019.

14. Philippe Duchon, Philippe Flajolet, Guy Louchard, and Gilles Schaeffer. Boltzmann samplers for the random generation of combinatorial structures. Combinatorics, Probability and Computing, 13(4-5):577-625, 2004.

15. Gidon Ernst, Sean Sedwards, Zhenya Zhang, and Ichiro Hasuo. Fast falsification of hybrid systems using probabilistically adaptive input. In David Parker and Verena Wolf, editors, Quantitative Evaluation of Systems, 16th International Conference, QEST 2019, Glasgow, UK, September 10-12, 2019, Proceedings, volume 11785 of Lecture Notes in Computer Science, pages 165-181. Springer, 2019.

16. Georgios E. Fainekos and George J. Pappas. Robustness of temporal logic specifications for continuous-time signals. Theor. Comput. Sci., 410(42):4262-4291, 2009.

17. Jason Ghidella and Pieter Mosterman. Requirements-based testing in aircraft control design. In AIAA Modeling and Simulation Technologies Conference and Exhibit, page 5886, 2005.

18. Ary L Goldberger, Luis AN Amaral, Leon Glass, Jeffrey M Hausdorff, Plamen Ch Ivanov, Roger G Mark, Joseph E Mietus, George B Moody, Chung-Kang Peng, and H Eugene Stanley. Physiobank, physiotoolkit, and physionet: components of a new research resource for complex physiologic signals. Circulation, 101(23):e215-e220, 2000.

19. Radu Grosu and Scott A. Smolka. Monte carlo model checking. In Nicolas Halbwachs and Lenore D. Zuck, editors, Tools and Algorithms for the Construction and Analysis of Systems, pages 271-286, Berlin, Heidelberg, 2005. Springer Berlin Heidelberg.

20. Rahul Gupta, Shubham Sharma, Subhajit Roy, and Kuldeep S. Meel. Waps: Weighted and projected sampling. In Tomáš Vojnar and Lijun Zhang, editors, Tools and Algorithms for the Construction and Analysis of Systems, pages 59-76, Cham, 2019. Springer International Publishing.

21. Dejan Jovanovic and Leonardo de Moura. Solving non-linear arithmetic. ACM Commun. Comput. Algebra, 46(3/4):104-105, 2012.

22. J. Kennedy and R. Eberhart. Particle swarm optimization. In Proceedings of ICNN'95 - International Conference on Neural Networks, volume 4, pages 19421948 vol.4, 1995.

23. Seksan Kiatsupaibul, Robert L. Smith, and Zelda B. Zabinsky. An analysis of a variation of hit-and-run for uniform sampling from general regions. ACM Trans. Model. Comput. Simul., 21(3):16:1-16:11, 2011.

24. N. Kitchen and A. Kuehlmann. Stimulus generation for constrained random simulation. In 2007 IEEE/ACM International Conference on Computer-Aided Design, pages 258-265, 2007.

25. Kim G. Larsen and Axel Legay. Statistical model checking past, present, and future. In Tiziana Margaria and Bernhard Steffen, editors, Leveraging Applications of Formal Methods, Verification and Validation. Specialized Techniques and Applications, pages 135-142, Berlin, Heidelberg, 2014. Springer Berlin Heidelberg. 
26. Axel Legay, Benoît Delahaye, and Saddek Bensalem. Statistical model checking: An overview. In Howard Barringer, Ylies Falcone, Bernd Finkbeiner, Klaus Havelund, Insup Lee, Gordon Pace, Grigore Roşu, Oleg Sokolsky, and Nikolai Tillmann, editors, Runtime Verification, pages 122-135, Berlin, Heidelberg, 2010. Springer Berlin Heidelberg.

27. Axel Legay, Anna Lukina, Louis Marie Traonouez, Junxing Yang, Scott A. Smolka, and Radu Grosu. Statistical model checking. In Bernhard Steffen and Gerhard Woeginger, editors, Computing and Software Science: State of the Art and Perspectives, pages 478-504, Cham, 2019. Springer International Publishing.

28. László Lovász. Hit-and-run mixes fast. Math. Program., 86:443-461, 1999.

29. Oded Maler and Dejan Nickovic. Monitoring temporal properties of continuous signals. In Formal Techniques, Modelling and Analysis of Timed and Fault-Tolerant Systems, Joint International Conferences on Formal Modelling and Analysis of Timed Systems, FORMATS 2004 and Formal Techniques in Real-Time and FaultTolerant Systems, FTRTFT 2004, Grenoble, France, September 22-24, 2004, Proceedings, pages 152-166, 2004.

30. Radford M. Neal. Slice sampling. Ann. Statist., 31(3):705-767, 062003.

31. Truong Nghiem, Sriram Sankaranarayanan, Georgios E. Fainekos, Franjo Ivancic, Aarti Gupta, and George J. Pappas. Monte-carlo techniques for falsification of temporal properties of non-linear hybrid systems. In Proceedings of the 13th ACM International Conference on Hybrid Systems: Computation and Control, HSCC 2010, Stockholm, Sweden, April 12-15, 2010, pages 211-220, 2010.

32. Dejan Nickovic, Xin Qin, Thomas Ferrère, Cristinel Mateis, and Jyotirmoy V. Deshmukh. Shape expressions for specifying and extracting signal features. In Runtime Verification - 19th International Conference, RV 2019, Porto, Portugal, October 8-11, 2019, Proceedings, pages 292-309, 2019.

33. Johan Oudinet, Alain Denise, Marie-Claude Gaudel, Richard Lassaigne, and Sylvain Peyronnet. Uniform Monte-Carlo Model Checking. In FASE 2011, Saarbrücken, Germany, 2011.

34. Burcu Kulahcioglu Ozkan, Rupak Majumdar, Filip Niksic, Mitra Tabaei Befrouei, and Georg Weissenbacher. Randomized testing of distributed systems with probabilistic guarantees. Proceedings of the ACM on Programming Languages, 2(OOPSLA), 2018.

35. Angela Pappagallo, Annalisa Massini, and Enrico Tronci. Monte carlo based statistical model checking of cyber-physical systems: A review. Information 11, 12:588, 2020.

36. Pavithra Prabhakar, Ratan Lal, and James Kapinski. Automatic trace generation for signal temporal logic. In 2018 IEEE Real-Time Systems Symposium, RTSS 2018, Nashville, TN, USA, December 11-14, 2018, pages 208-217. IEEE Computer Society, 2018.

37. Chotirat Ann Ratanamahatana, Jessica Lin, Dimitrios Gunopulos, Eamonn J. Keogh, Michail Vlachos, and Gautam Das. Mining time series data. In Data Mining and Knowledge Discovery Handbook, 2nd ed, pages 1049-1077. Springer, 2010.

38. S-Taliro tool. https://sites.google.com/a/asu.edu/s-taliro/references.

39. Robert L. Smith. Efficient monte carlo procedures for generating points uniformly distributed over bounded regions. Oper. Res., 32(6):1296-1308, 1984.

40. Jun Yuan, Carl Pixley, and Adnan Aziz. Constraint-based verification. Springer Science \& Business Media, 2006. 
41. Zhenya Zhang, Paolo Arcaini, and Ichiro Hasuo. Constraining counterexamples in hybrid system falsification: Penalty-based approaches. In Ritchie Lee, Susmit Jha, and Anastasia Mavridou, editors, NASA Formal Methods - 12th International Symposium, NFM 2020, Moffett Field, CA, USA, May 11-15, 2020, Proceedings, volume 12229 of Lecture Notes in Computer Science, pages 401-419. Springer, 2020 .

\section{A Evaluation of Hit-and-Run}

We evaluate different hit-and-run variants presented in Section 3 . In our experiments, classical rejection sampling, denoted by rs, is used as our baseline.

Our specification is defined by the $n$-dimensional hyper-ring: $\chi=x_{1}^{2}+\ldots+$ $x_{n}^{2}<c_{1}^{2} \wedge x_{1}^{2}+\ldots+x_{n}^{2}>c_{2}^{2}$ where $c_{1}>c_{2}$, and the hyper-box constraint $\psi$ embodying the hyper-ring: $\psi=\bigwedge_{i=1}^{n} x_{i}>-c \wedge x_{i}<c$ where $c \geq c_{1}$. This hyper-ring specification has several interesting characteristics: (1) it is polynomial, (2) it is non-convex, (3) it can be instantiated to an arbitrary number of dimensions, and (4) it can be made arbitrarily "thin".

We first study hit-and-run variants with respect to the number of dimensions. For this experiment, we generate 100 samples from the set defined by $\chi \wedge \psi$, where $c=c_{1}=1$ and $c_{2}=0$. We vary the number of dimensions in the specification and repeat each experiment 5 times. Figure 9 summarizes the results.

We first remark that classical rejection sampling does not scale beyond 3 dimensions. Also, the hit-and-run variants that use search fail to find an initial sample in more than 10 dimensions. The use of the SMT solver helps to efficiently find an initial sample and enables scaling to 50 dimensions. We finally observe that the shrinking mechanism allows halving the computation time (and sampling 100 points in a 100-dimensional ball in 12s) and considerably improving the acceptance rates, clearly outperforming the "vanilla" version of hit-and-run.
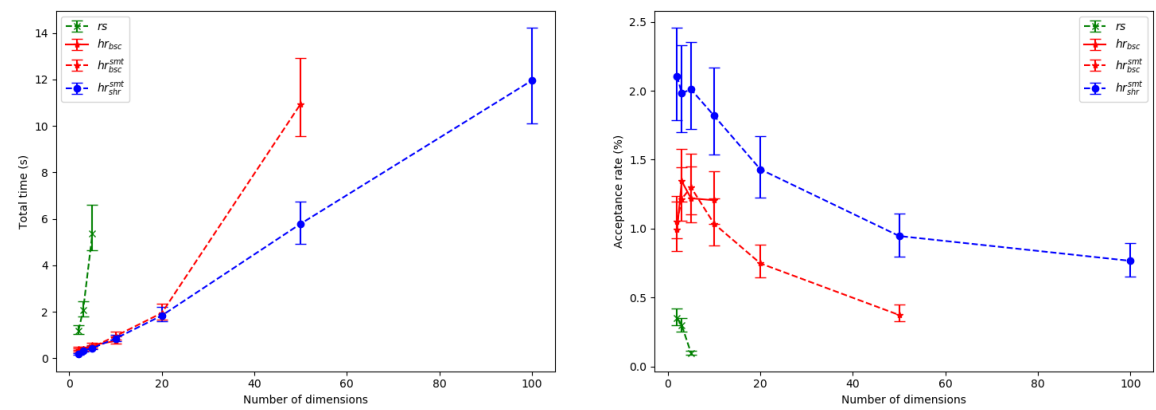

Fig. 9: Performance of hit-and-run in number of dimensions: (1) computation time (left) and (2) acceptance rate (right). 
In the next experiment, we study the distribution of samples that it generates for hyper-rings of various thickness. In Figure 10, we visually compare the evolution of the sample distributions generated from a constraint defining a 2-dimensional ring with $c_{1}=1$ and $c_{2}=0.9$ by rejection sampling and by hitand-run, where we take snapshots after generating 10, 50, 200 and 1000 samples.
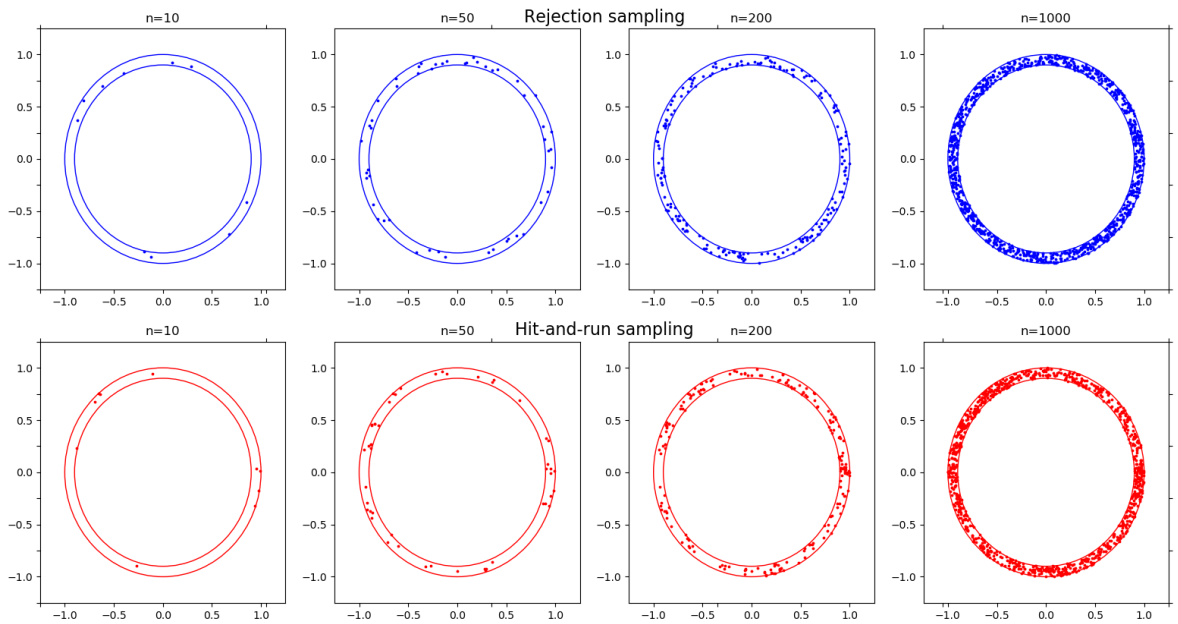

Fig. 10: Evolution of sample distribution generated by hit-and-run compared to that by rejection sampling.

We conduct two more experiments that evaluate the acceptance rate of hitand-run with respect to: (1) the thickness of the hyper-ring, and (2) the size of the bounding hyper-box. In these experiments, we consider a 3-dimensional hyper-ring, in which we sample 1000 times. We repeat each experiment 5 times.

We vary the hyper-ring thickness by fixing the constants $c$ and $c_{1}$ to 1 , and by varying the value of $c_{2}$ among $0.5,0.75,0.9$ and 0.99 . Figure 11 (left) summarizes the results. We can first observe that the thinner the hyper-ring is, the closer the acceptance rate of the basic hit-and-run algorithm comes to the standard rejection sampling. On the other hand, the shrinking mechanism allows to retain reasonable acceptance rates even for quite thin constraints (more than $10 \%$ acceptance rate when $c_{1}=1$ and $c_{2}=0.99$ ).

We finally fix the constants $c_{1}$ to 1 and $c_{2}$ to 0.9 and vary the size of hyperrectangles (by changing the value of $c$ ) among 1, 5, 10 and 20. The results of this experiment are summarized in Figure 11 (right). We first observe that acceptance rate of the rejection sampling procedure when $c=5$ is only around $0.06 \%$. We can also observe a similar trend of the acceptance rate dropping for the two hit-and-run procedures when the bounding box becomes large. We no- 
tice that the hit-and-run method with shrinking does perform better by an order of magnitude and maintains an acceptance rate above $10 \%$ when $c=20$.
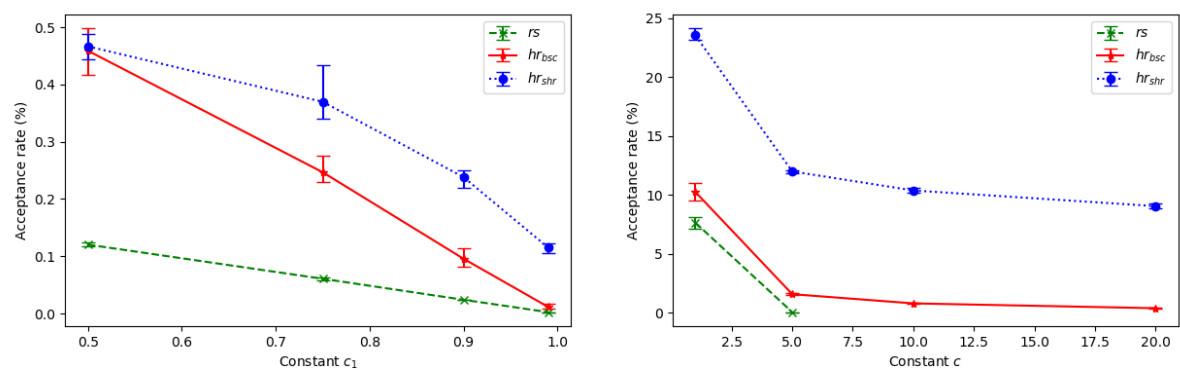

Fig. 11: Acceptance rate with respect to the thickness of the ring (left) and to the size of the bounding box (right). 